\title{
Stark effect in the nonuniform field and its influence on the fine structure of Rydberg blockade
}

\author{
Yurii V Dumin ${ }^{1,2,3}$ \\ ${ }^{1}$ Max Planck Institute for the Physics of Complex Systems (MPIPKS), \\ Noethnitzer Str. 38, 01187 Dresden, Germany \\ 2 Sternberg Astronomical Institute (GAISh) of Lomonosov Moscow State University, \\ Universitetski prosp. 13, 119992 Moscow, Russia \\ 3 Space Research Institute (IKI) of Russian Academy of Sciences, \\ Profsoyuznaya str. 84/32, 117997 Moscow, Russia \\ E-mail: dumin@pks.mpg.de, dumin@yahoo.com
}

\begin{abstract}
Splitting the energy levels of a hydrogen-like atom by the electric field nonuniform at the atomic scale is studied. This situation is important for the multilevel treatment of the phenomenon of Rydberg blockade [Yu.V. Dumin, J. Phys. B, v. 47, p. 175502 (2014)]. An explicit formula for the energy levels is derived. A typical value of the energy shift by the electric field gradient turns out to be proportional to the 4th power of the principal quantum number (i.e., the square of atomic size), as would be expected from a qualitative consideration. Finally, the fine spatial structure of the Rydberg blockade is analyzed when the electric-field-gradient term plays the dominant role, and the results are confronted with the experimental data.
\end{abstract}

PACS numbers: $32.60 .+\mathrm{i}, 32.80 . \mathrm{Ee}$

Submitted to: J. Phys. B: At. Mol. Opt. Phys. 


\section{Introduction}

Splitting the atomic energy levels by an external electric field (Stark effect) is studied both experimentally and theoretically for almost a century (e.g., reviews [1, 2]). However, almost all previous theoretical treatments were performed in the approximation of the uniform external field $\mathcal{E}_{0}$. This is not surprising because, until recently, it was difficult to imagine that the electric field can be variable at the atomic scale.

The only work beyond this approximation, familiar to us, was done in 1970 by Bekenstein and Krieger [3], who assumed that the substantially nonuniform electric fields could be produced just by the neighboring atoms in a sufficiently dense gas. So, the corresponding Stark effect would result in the specific broadening of the spectral lines. Unfortunately, deriving the parameters of the nonuniform Stark effect from the line broadening is a quite indirect and ambiguous procedure; so that this idea did not attract a sufficient attention at that time.

However, the situation changed in the recent decade in the context of the so-called Rydberg blockade, which was discussed, at first, theoretically [4] and then confirmed experimentally [5, 6, 7]. Briefly speaking, this effect occurs when the electric field of an initially-excited Rydberg atom disturbs the energy levels of the neighboring groundstate atoms, so that their excitation by a narrow-band laser irradiation to the same quantum state becomes impossible (because this state goes out of the resonance); a pictorial illustration can be found below in figure 1 .

Although the phenomenon of Rydberg blockade is usually considered in the approximation of the selected essential states, a reasonable alternative treatment can be based on the consideration of Stark effect produced by an already excited Rydberg atom on the surrounding atoms. A particular advantage of such an approach is that it can reveal complex spatial structure of the blockade zone, namely, a sequence of intermittent co-centric shells where the possibility of excitation becomes blocked and unblocked again [8].

However, usage of the standard formulas for Stark effect seems to be questionable in the above-mentioned situation: the characteristic interatomic separation is only a few times greater than a typical size of the Rydberg atom and, therefore, the electric field becomes substantially nonuniform at the atomic scale. So, it is desirable to perform the corresponding analysis taking into account Stark splitting by the nonuniform external field.

Below, in section 2, we first briefly remind the basic mathematical formalism for dealing with Stark effect by the quantization in parabolic coordinates and then apply the perturbation technique to the case of the nonuniform electric field. (As distinct from the above-cited paper [3], our approach will not use the WKB approximation.) At last, in section 3, we study the Rydberg blockade in the situation when it is produced mostly by the gradient term of the Stark splitting and discuss the respective observational consequences. 


\section{Stark effect in the nonuniform external field}

\subsection{Basic formulas for the unperturbed Coulomb's problem}

The starting point of our consideration is Schroedinger equation for an electron in the Coulomb's field of the nucleus with charge $Z$, located in the origin of coordinates, and the external electric field $\mathcal{E}$, directed along $z$-axis (all formulas are written in the atomic units):

$$
\left[-\frac{1}{2} \triangle-\frac{Z}{r}+\delta U(z)\right] \psi=E \psi
$$

where $\delta U$ is perturbation of the atomic potential energy by the electric field

$$
\mathcal{E}(z)=\mathcal{E}_{0}+\left(\frac{d \mathcal{E}}{d z}\right)_{0} z+\ldots,
$$

where subscript 0 denotes the corresponding values in the origin of coordinates. So, the perturbation in explicit form is

$$
\delta U(z)=\mathcal{E}_{0} z+\frac{1}{2}\left(\frac{d \mathcal{E}}{d z}\right)_{0} z^{2}+\ldots
$$

(It is written with a plus sign because it refers to the negatively-charged electron.)

If $\delta U \equiv 0$, then equation (1) can be solved exactly in the parabolic coordinates in terms of either the confluent hypergeometric functions (e.g., textbook [9], §37) or the associated Laguerre functions $L_{\lambda}^{\mu}$ (monograph [1], section 6). We prefer to use the second option, so the solution will take the form:

$$
\begin{aligned}
& \psi_{n_{1} n_{2} m}^{(0)}(\xi, \eta, \varphi)=\frac{e^{ \pm i m \varphi}}{\sqrt{\pi n}} \\
& \quad \times \frac{\left(n_{1} !\right)^{1 / 2}}{\left(\left(n_{1}+m\right) !\right)^{3 / 2}} \frac{\left(n_{2} !\right)^{1 / 2}}{\left(\left(n_{2}+m\right) !\right)^{3 / 2}} \varepsilon^{m+\frac{3}{2}} \\
& \quad \times e^{-\varepsilon(\xi+\eta) / 2}(\xi \eta)^{m / 2} L_{n_{1}+m}^{m}(\varepsilon \xi) L_{n_{2}+m}^{m}(\varepsilon \eta) .
\end{aligned}
$$

Here, $\varepsilon=Z / n, \varphi$ is the azimuthal angle; and $\xi, \eta$ are the parabolic coordinates, related to the Cartesian coordinates by the standard formulas:

$$
\begin{aligned}
& \xi=r+z, \\
& \eta=r-z, \\
& \varphi=\arctan (x / y), \\
& r=\sqrt{x^{2}+y^{2}+z^{2}}=\frac{1}{2}(\xi+\eta) ;
\end{aligned}
$$

or vice versa:

$$
\begin{aligned}
& x=\sqrt{\xi \eta} \cos \varphi, \\
& y=\sqrt{\xi \eta} \sin \varphi, \\
& z=\frac{1}{2}(\xi-\eta),
\end{aligned}
$$


where

$$
\xi, \eta \in[0,+\infty), \quad \varphi \in[0,2 \pi] .
$$

The elementary length and volume in the parabolic coordinates are

$$
\begin{aligned}
& d l^{2}=\frac{\xi+\eta}{4 \xi} d \xi^{2}+\frac{\xi+\eta}{4 \eta} d \eta^{2}+\xi \eta d \varphi^{2}, \\
& d V=\frac{\xi+\eta}{4} d \xi d \eta d \varphi .
\end{aligned}
$$

The eigenvalues $E^{(0)}$ of the unperturbed equation (11) are associated with the principal quantum number $n$ by the usual formula:

$$
n=\frac{1}{\sqrt{-2 E^{(0)}}}, \quad n=1,2,3, \ldots ;
$$

while the principal quantum number is related to the parabolic quantum numbers $n_{1}$ and $n_{2}$ (which are the nonnegative integers) and the magnetic quantum number $m$ as

$$
n=n_{1}+n_{2}+m+1,
$$

where $m$ is assumed to be positive or zero, since the plus/minus sign was already written explicitly in (4).

\subsection{Perturbation by the external field}

Let us find eigenvalues of the Schroedinger equation (1) perturbed by the nonuniform external electric field (2). As was already mentioned in the Introduction, a similar problem was treated long time ago by Bekenstein and Krieger [3], who developed a quite sophisticated mathematical technique based on the WKB approximation. So, our aim here is to perform the same calculations beyond the above-mentioned approximation and, thereby, to check and refine the earlier results.

If $(d \mathcal{E} / d z)_{0}=0$, i.e., the external electric field is uniform, then the first-order correction to the energy levels is well known [1, 2, 9]:

$$
(\delta E)_{\mathrm{unif}}=\frac{3}{2} \mathcal{E} n\left(n_{1}-n_{2}\right) .
$$

In the more general case, when $(d \mathcal{E} / d z)_{0} \neq 0$, i.e., the electric field gradient is present, the perturbation of the energy levels $\delta E$ is given in the first approximation by the diagonal matrix element of operator (3) with respect to the unperturbed states (4):

$$
\delta E=\iiint\left|\psi_{n_{1} n_{2} m}\right|^{2}(\delta U) d V
$$

(for rigorous mathematical justification of using the first-order perturbation theory in the nonuniform Stark problem, see [3] and references therein).

Substituting expression (3) into (14) and using the properties of parabolic coordinates (17c) and (10), we get: 


$$
\begin{aligned}
\delta E=\frac{1}{8} \int_{0}^{\infty} \int_{0}^{\infty} \int_{0}^{2 \pi}\left|\psi_{n_{1} n_{2} m}\right|^{2}\left[\mathcal{E}_{0}\left(\xi^{2}-\eta^{2}\right)\right. \\
\left.+\frac{1}{4}\left(\frac{d \mathcal{E}}{d z}\right)_{0}\left(\xi^{2}-\eta^{2}\right)(\xi-\eta)\right] d \varphi d \xi d \eta
\end{aligned}
$$

Then, the total energy of the split sublevels can be written as

$$
E_{n_{1} n_{2} m}=-\frac{1}{2} \frac{Z^{2}}{n^{2}}+(\delta E)_{n_{1} n_{2} m}
$$

where

$$
\begin{aligned}
& (\delta E)_{n_{1} n_{2} m}=\frac{1}{4 n} \frac{n_{1} !}{\left(n_{1}+m\right) !} \frac{n_{2} !}{\left(n_{2}+m\right) !} \\
& \quad \times\left\{\frac{\mathcal{E}_{0}}{\varepsilon}\left[J_{n_{1}+m, m}^{(2)} J_{n_{2}+m, m}^{(0)}-J_{n_{1}+m, m}^{(0)} J_{n_{2}+m, m}^{(2)}\right]\right. \\
& \quad+\frac{(d \mathcal{E} / d z)_{0}}{4 \varepsilon^{2}}\left[J_{n_{1}+m, m}^{(3)} J_{n_{2}+m, m}^{(0)}+J_{n_{1}+m, m}^{(0)} J_{n_{2}+m, m}^{(3)}\right. \\
& \left.\left.\quad-J_{n_{1}+m, m}^{(2)} J_{n_{2}+m, m}^{(1)}-J_{n_{1}+m, m}^{(1)} J_{n_{2}+m, m}^{(2)}\right]\right\} .
\end{aligned}
$$

Here, the integrals $J_{\lambda \mu}^{(\sigma)}$ are defined by the standard way as

$$
J_{\lambda \mu}^{(\sigma)}=\frac{1}{(\lambda !)^{2}} \int_{0}^{\infty} e^{-\rho} \rho^{\mu+\sigma}\left[L_{\lambda}^{\mu}(\rho)\right]^{2} d \rho .
$$

Their explicit calculation gives the following expression in terms of the binomial coefficients (e.g., [1], section 3):

$$
\begin{aligned}
J_{\lambda \mu}^{(\sigma)} & =(-1)^{\sigma} \frac{\lambda !}{(\lambda-\mu) !} \sigma ! \\
& \times \sum_{\beta=0}^{\sigma}(-1)^{\beta}\left(\begin{array}{c}
\sigma \\
\beta
\end{array}\right)\left(\begin{array}{c}
\lambda+\beta \\
\sigma
\end{array}\right)\left(\begin{array}{c}
\lambda+\beta-\mu \\
\sigma
\end{array}\right) .
\end{aligned}
$$

In particular, the integrals required for us are

$$
\begin{aligned}
J_{k+m, m}^{(0)}= & \frac{(k+m) !}{k !}, \\
J_{k+m, m}^{(1)}= & \frac{(k+m) !}{k !}(2 k+m+1), \\
J_{k+m, m}^{(2)}= & \frac{(k+m) !}{k !}\left[(k+m)^{2}+(k+m)(4 k+3)+\left(k^{2}+3 k+2\right)\right], \\
J_{k+m, m}^{(3)}= & \frac{(k+m) !}{k !}\left(20 k^{3}+30 k^{2}+22 k+30 k^{2} m+12 k m^{2}+30 k m\right. \\
& \left.\quad+m^{3}+6 m^{2}+11 m+6\right) .
\end{aligned}
$$


After the substitution of (20a)-(20d) into (17) and (16), the final result for splitting the energy levels can be written in a quite compact form as

$$
\begin{aligned}
& E_{n_{1} n_{2} m}=-\frac{1}{2} \frac{Z^{2}}{n^{2}}+\frac{3}{2} \mathcal{E}_{0} \frac{n}{Z}\left(n_{1}-n_{2}\right) \\
& +\frac{1}{4}\left(\frac{d \mathcal{E}}{d z}\right)_{0} \frac{n^{2}}{Z^{2}}\left[5\left(n_{1}-n_{2}\right)^{2}+2\left(n_{1} n_{2}+1\right)+(n-m) m+n_{1}+n_{2}\right],
\end{aligned}
$$

where $n=n_{1}+n_{2}+m+1\left(n_{1} \geq 0, n_{2} \geq 0, m \geq 0\right)$. Here, the first term represents the energy of an unperturbed hydrogen-like atom, the second term is the well-known expression for linear Stark effect in the uniform external field [1, 2, and the third (gradient) term is the required correction for nonuniformity. To avoid misunderstanding, let us emphasize that this gradient term should not be mixed with the higher-order corrections with respect to the electric field amplitude $\mathcal{E}_{0}$, which were widely discussed in the previous literature (e.g., the procedure for obtaining the corrections of an arbitrary order can be found in [10]).

\subsection{Qualitative features}

Let us briefly discuss a qualitative behavior of the gradient term. First of all, it should be noticed that the electric field gradient partially lifts a degeneracy of the energy levels with respect to the magnetic quantum number $m$ (apart from the plus/minus sign in formula (44) ) already in the first order of the perturbation theory, while in the uniform field this is possible only in the second order [1, 2].

Next, as is known, the linear Stark effect in a uniform field is roughly proportional to $n^{2} \mathcal{E}_{0}$, which is just a typical difference of the electric potential across the atom. Besides, this quantity can be substantially reduced under appropriate choice of the quantum numbers, e.g., when the atom is approximately symmetric $\left(n_{1} \approx n_{2}\right)$.

The gradient term exhibits a quite similar behavior: as follows from (21), its characteristic magnitude is $(d \mathcal{E} / d z)_{0} n^{4}$, which represents again the typical potential difference across the atom. This value can also be substantially reduced at the appropriate combination of the quantum numbers. However, the sum in the square brackets always remains positive, so that the sign of the resulting effect is completely determined by the sign of the derivative $(d \mathcal{E} / d z)_{0}$.

At last, let us make a few remarks about the application of our results to other Rydberg atoms than hydrogen, namely, with presence of the quantum defects. A detailed study of this issue, evidently, requires a separate paper. However, it can be reasonably believed that difference in the gradient term of the Stark effect between the purely hydrogenic and hydrogen-like atoms will not be so dramatic as in the case of the uniform field. Really, the crucial feature of Stark effect in the hydrogen-like atoms with non-degenerate spectra is that it is quadratic with respect to the field amplitude $\mathcal{E}_{0}$ : the first-order correction to energy $\mathcal{E}_{0}\langle\psi|z| \psi\rangle$ vanishes because the wavefunction $\mid \psi>$ possesses a definite parity under the transformation $z \rightarrow-z$, while the operator $z$ changes its sign. So, the matrix element $\langle\psi|z| \psi\rangle$ can be nonzero only 
for pure hydrogen, where the energy spectrum is degenerate and, therefore, $|\psi\rangle$ can be a superposition of states with various parities.

On the other hand, when we calculate the gradient correction, which is proportional to $z^{2}$, the difference should not be so drastic: since operator $z^{2}$ does not change its sign under the transformation $z \rightarrow-z$, the first-order correction $\left.(1 / 2)(d \mathcal{E} / d z)_{0}<\psi\left|z^{2}\right| \psi\right\rangle$, in general, will not be equal to zero even in the non-degenerate state with a certain parity. Therefore, there should be not so much difference between the purely hydrogenic and hydrogen-like atoms in the case of the nonuniform-field Stark effect.

\subsection{Comparison with the earlier calculations}

A convenient benchmark for testing our calculations are the results by Bekenstein and Krieger (BK) [3], which were derived in the WKB approximation. They can be rewritten in our designations as:

$$
\begin{aligned}
& \left.\delta E^{(\mathrm{BK})}\right|_{\mathcal{E}_{0}=0}=\frac{1}{4}\left(\frac{\partial \mathcal{E}_{z}}{\partial z}\right)_{0} \frac{n^{2}}{Z^{2}} \\
& \quad \times\left[5\left(n_{1}-n_{2}\right)^{2}-2\left(2 n_{1} n_{2}+n_{1}+n_{2}\right)\right. \\
& \quad+m(m-2 n)] \\
& \quad+\frac{3}{4}\left[\left(\frac{\partial \mathcal{E}_{x}}{\partial x}\right)_{0}-\left(\frac{\partial \mathcal{E}_{y}}{\partial y}\right)_{0}\right]_{m} \frac{n^{2}}{Z^{2}}\left(n_{1}+1\right)\left(n_{2}+1\right),
\end{aligned}
$$

where

$$
\epsilon_{m}=\left\{\begin{array}{rll} 
\pm 1 & \text { at } & m=1, \\
0 & \text { at } & m \neq 1,
\end{array}\right.
$$

and the plus/minus sign corresponds to the sign of $m$ in the wavefunction (4).

The second term in formula (22) vanishes for any axially-symmetric field, when $\partial \mathcal{E}_{x} / \partial x=\partial \mathcal{E}_{y} / \partial y$. In particular, the respective components of the electric-field-gradient tensor were ignored in our initial formulation of the problem. So, only the first term of (22) should be confronted with our formula (21).

It is seen that these two expressions, in general, are somewhat different from each other. However, the first term in the square brackets is the same. So, in the case most important from the experimental point of view, when the magnetic quantum number $m$ is small and the atomic state is strongly asymmetric (i.e., one of the parabolic quantum numbers, $n_{1}$ or $n_{2}$, is close to zero and another is approximately equal to the principal quantum number $n \gg 1$ ), both formulas give the same result: $(5 / 4)(d \mathcal{E} / d z)_{0}\left(n^{4} / Z^{2}\right)$.

On the other hand, the WKB approximation (22) substantially deviates from the exact solution (21) for the symmetric states, where $n_{1} \approx n_{2}$. Moreover, this approximation leads to the conclusion that the gradient-term Stark effect can change its sign in such states, which is very suspicious from the physical point of view. Besides, as follows from these formulas, dependence on the magnetic quantum number $m$ in the WKB approximation is inadequate. 
So, the analysis performed in section 2.2 enabled us to improve substantially the previously-known formula for Stark effect in the nonuniform field. As will be seen below in section 3.1, the established properties (in particular, the impossibility to change the sign of the total effect) lead to important qualitative features in the pattern of Rydberg blockade.

\section{Rydberg blockade}

As was already mentioned in the Introduction, the most interesting application of Stark effect in the nonuniform field is a multi-level treatment of the Rydberg blockade, which was performed for the case of a uniform field in our previous article 8. The same analysis taking into account all terms in formula (21) is quite straightforward but cumbersome and, therefore, requires a separate paper. So, we shall restrict our consideration here by the case when the gradient term plays a dominant role. From the physical point of view, this situation assumes a strong electric-field gradient $(d \mathcal{E} / d z)_{0}$ and/or very large values of the principal quantum number $n$.

\subsection{Blockade by the gradient term}

We shall consider below a neutral hydrogen-like atom, i.e., take $Z=1$. Besides, since the experiments on Rydberg blockade are typically performed with atoms possessing small values of the magnetic quantum number $(m=0,1,2)$, it is reasonable to neglect the terms with $m$ in formula (21). Within the same accuracy, we can discard also the terms on the order of unity as compared to $n$. At last, expressing $n_{2}$ in terms of $n$ and $n_{1}$, the energy of the split sublevels is written as

$$
E_{n n_{1}} \approx-\frac{1}{2} \frac{1}{n^{2}}+\frac{1}{4}\left(\frac{d \mathcal{E}}{d z}\right)_{0} n^{2}\left[5 n^{2}-18 n_{1}\left(n-n_{1}\right)\right] .
$$

Next, it can be easily shown that the expression in square brackets is always positive and, as function of $n_{1}$, takes a maximum value at the boundaries of the domain of definition, for example, at $n_{1}=0$ (i.e., in the case of the most asymmetric atom). So, the energy of the most perturbed sublevel with principal quantum number $n$ takes the form:

$$
E_{n}^{(\max )}=-\frac{1}{2} \frac{1}{n^{2}}+\frac{5}{4}\left(\frac{d \mathcal{E}}{d z}\right)_{0} n^{4} .
$$

Following the same procedure as in our previous paper [8], just this sublevel will be used to estimate the characteristic parameters of the Rydberg blockade zone.

Similarly to the above-cited article, we assume that the dipolar electric field produced by an already excited Rydberg atom, located in the origin of coordinates, is

$$
\mathcal{E}(r)=\frac{C n^{2}}{r^{3}},
$$


where $C$ is a dimensionless coefficient on the order of unity, which can be both positive and negative depending on the dipole orientation. (Since the atomic system of units is used everywhere in the present work, we shall not write tildes as in the previous paper [8].)

Without going into details of the angular dependence, the derivative of the electric field can be roughly estimated as

$$
\left(\frac{d \mathcal{E}}{d z}\right)_{0} \approx \pm\left(\frac{d \mathcal{E}}{d r}\right)_{0}=\mp \frac{3 C n^{2}}{r^{4}} .
$$

Here, the upper and lower signs correspond to the cases when the perturbed atom is located, respectively, in the positive and negative direction of $z$-axis; and subscript 0 refers, as in section 2, to the position of the atom experiencing the Stark effect.

To be specific, let us assume that the value of the derivative $(d \mathcal{E} / d z)_{0}$ is positive:

$$
\left(\frac{d \mathcal{E}}{d z}\right)_{0}=\frac{3|C| n^{2}}{r^{4}}
$$

so that expression (25) is reduced to

$$
E_{n}^{(\max )}=-\frac{1}{2} \frac{1}{n^{2}}+\frac{15|C|}{4} \frac{n^{6}}{r^{4}} .
$$

The entire Stark manifold is schematically drawn in figure 1. As distinct from the pattern for the uniform field [8], all the sublevels are shifted in the same direction (upwards if $(d \mathcal{E} / d z)_{0}>0$ or downwards if $\left.(d \mathcal{E} / d z)_{0}<0\right)$. Therefore, in the first case, only the levels with lower values of the principal quantum number $(|n-1\rangle,|n-2\rangle$, etc.) can be unblocked at the sufficiently small distances.

In the same way as in paper [8], the condition of Rydberg blockade of the basic level $|n\rangle$ at the distance $R_{\mathrm{b}}^{(n)}$ can be written as

$$
-\frac{1}{2} \frac{1}{n^{2}}+\frac{15|C|}{4} \frac{n^{6}}{\left(R_{\mathrm{b}}^{(n)}\right)^{4}}=-\frac{1}{2} \frac{1}{n^{2}}+\frac{1}{2} \Delta E ;
$$

so that

$$
\Delta E=\frac{15|C|}{2} \frac{n^{6}}{\left(R_{\mathrm{b}}^{(n)}\right)^{4}},
$$

where $\Delta E$ is the characteristic bandwidth of the exciting radiation.

Next, the condition of unblocking and subsequent blocking of the lower state $|n-1\rangle$ at the radii $R_{\mathrm{u}}^{(n-1)}$ and $R_{\mathrm{b}}^{(n-1)}$, respectively, takes the form:

$$
-\frac{1}{2} \frac{1}{(n-1)^{2}}+\frac{15|C|}{4} \frac{(n-1)^{6}}{\left(R_{\mathrm{u}, \mathrm{b}}^{(n-1)}\right)^{4}}=-\frac{1}{2} \frac{1}{n^{2}} \mp \frac{1}{2} \Delta E .
$$

Substituting here expression (31) and neglecting the terms on the order of $1 / n$ as compared to unity, the above formula is reduced to

$$
\frac{1}{\left(R_{\mathrm{u}, \mathrm{b}}^{(n-1)}\right)^{4}}=\frac{4}{15|C|} \frac{1}{n^{9}} \mp \frac{1}{\left(R_{\mathrm{b}}^{(n)}\right)^{4}} .
$$




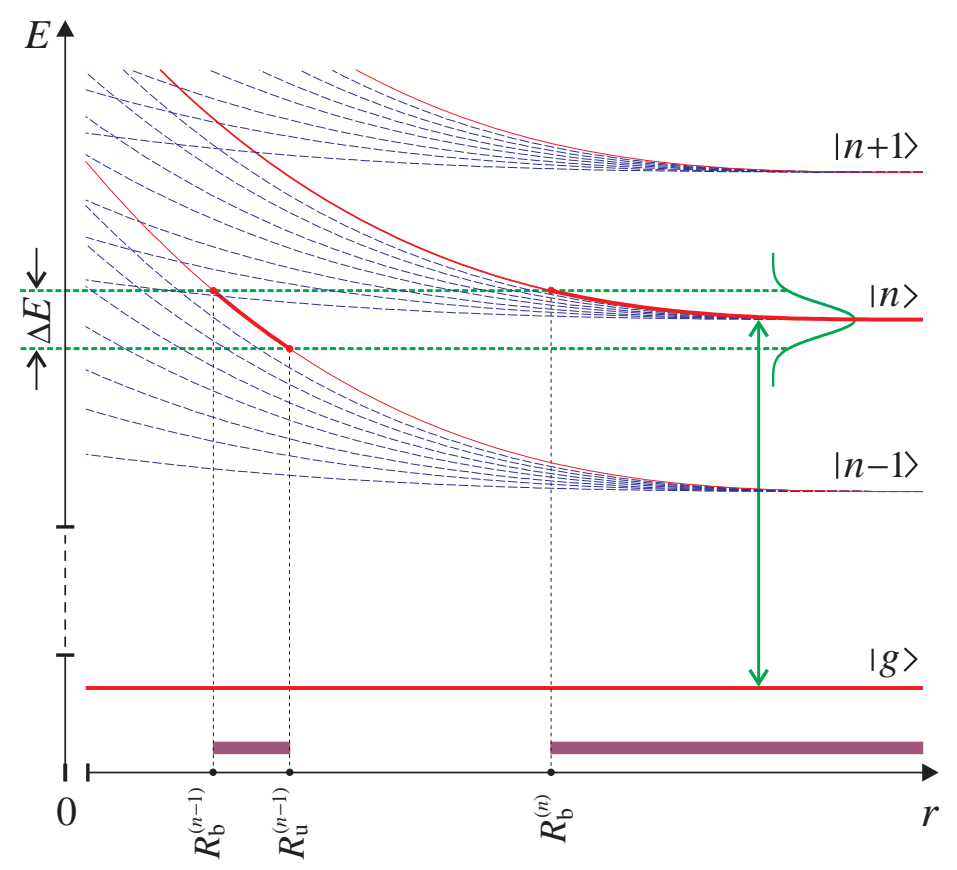

Figure 1. The multi-level pattern of Rydberg blockade caused by the gradient term. The energy levels of the atom under consideration are assumed to be split by the electric field of the central $(r=0)$ Rydberg-excited atom. The sublevels with maximal splitting are shown by solid (red) curves; and other ones, by the broken (blue) curves. The Rydberg excitation is possible only in the thick segments of the energy curves, located between the dotted (green) horizontal lines, which show a characteristic bandwidth of the exciting irradiation. The thick (violet) strips near the horizontal axis designate the corresponding intervals of radius: $\left[R_{\mathrm{b}}^{(n)},+\infty\right],\left[R_{\mathrm{b}}^{(n-1)}, R_{\mathrm{u}}^{(n-1)}\right]$, etc.

Therefore, we get finally:

$$
R_{\mathrm{u}, \mathrm{b}}^{(n-1)}=\left(\frac{15|C|}{4}\right)^{1 / 4} n^{9 / 4}\left\{1 \mp \frac{15|C|}{4} \frac{n^{9}}{\left(R_{\mathrm{b}}^{(n)}\right)^{4}}\right\}^{-1 / 4},
$$

where the minus/plus sign refers to the points where Rydberg excitation becomes unblocked and blocked again.

If the second term in braces in the right-hand side of formula (34) is small as compared to unity, then this expression can be reduced to

$$
R_{\mathrm{u}, \mathrm{b}}^{(n-1)} \approx\left(\frac{15|C|}{4}\right)^{1 / 4} n^{9 / 4}\left\{1 \pm \frac{15|C|}{16} \frac{n^{9}}{\left(R_{\mathrm{b}}^{(n)}\right)^{4}}\right\} .
$$

Consequently, a center of the additional excitation zone, corresponding to the interval $\left[R_{\mathrm{b}}^{(n-1)}, R_{\mathrm{u}}^{(n-1)}\right]$ in figure 1, is located at the distance

$$
R_{\mathrm{c}}^{(n-1)}=\left(\frac{15|C|}{4}\right)^{1 / 4} n^{9 / 4}
$$


from the already excited Rydberg atom; and its characteristic width equals

$$
\Delta R^{(n-1)}=\frac{1}{2}\left(\frac{15|C|}{4}\right)^{5 / 4} \frac{n^{45 / 4}}{\left(R_{\mathrm{b}}^{(n)}\right)^{4}} .
$$

Let us compare these expressions with the ones derived for the case of Rydberg blockade by the uniform field [8] (they will be designated by the subscript 'unif'):

$$
R_{\mathrm{c}, \text { unif }}^{(n-1)}=\left(\frac{3 C}{2}\right)^{1 / 3} n^{7 / 3}
$$

and

$$
\Delta R_{\text {unif }}^{(n-1)}=\left(\frac{3 C^{4}}{2}\right)^{1 / 3} \frac{n^{28 / 3}}{\left(R_{\mathrm{b}}^{(n)}\right)^{3}} .
$$

It is quite surprising that positions of the additional excitation zone in both cases are almost the same: the exponents of the principal quantum number $n$ in formulas (36) and (38) are very similar to each other, while the numerical coefficients are close to unity.

Finally, it should be mentioned that the patterns of Rydberg blockade presented in figure 1 of this paper, as well as in figure 1 of our previous article [8] involve a lot of intersections of the energetic levels with each other. Generally speaking, this will result in the level-anticrossing effects and mixing between the states of different quantum numbers, e.g., as discussed in [11]. However, all such phenomena are not so important in the context of Rydberg blockade. The crucial item for our consideration is the presence (or absence) of an allowed electronic state in the energy band of the exciting radiation. The level-anticrossing effects as well as the exact identification of the resulting energetic states are of secondary importance here. Of course, they should be taken into consideration in a more careful treatment of the Rydberg blockade, involving the calculation of probabilities of the corresponding transitions.

\subsection{Comparison with the experimental data}

To get some numerical estimates, let us use the parameters of experiment [12], which seems to be the most detailed spatially-resolved study of the Rydberg blockade available by now. In this case, $n=43$ and $R_{\mathrm{b}}^{(n)} \approx 4 \mu \mathrm{m} \approx 8 \times 10^{4}$ a.u. Then, both formulas (36) and (38) give $R_{\mathrm{c}}^{(n-1)} \approx 6 \times 10^{3}$ a.u. $\approx 0.3 \mu \mathrm{m}$. It is interesting that this value corresponds very well to the position of the additional unexpected peak in the pair correlation function of Rydberg atoms depicted in figure 3a of the above-cited paper, which is partially reproduced in our figure 2, Such a peak is evidently impossible in the "standard" model of Rydberg blockade, which takes into account only the states with the same value of the principal quantum number (the corresponding theoretical correlation function is drawn by the solid curve). So, this striking deviation might be associated just with formation of the additional unblocked zone for a neighboring value of the principal quantum number (see interval $\left[R_{\mathrm{b}}^{(n-1)}, R_{\mathrm{u}}^{(n-1)}\right]$ in figure 1) rather than caused by imperfections of the measurement procedures, as was originally suggested by the experimentalists. 


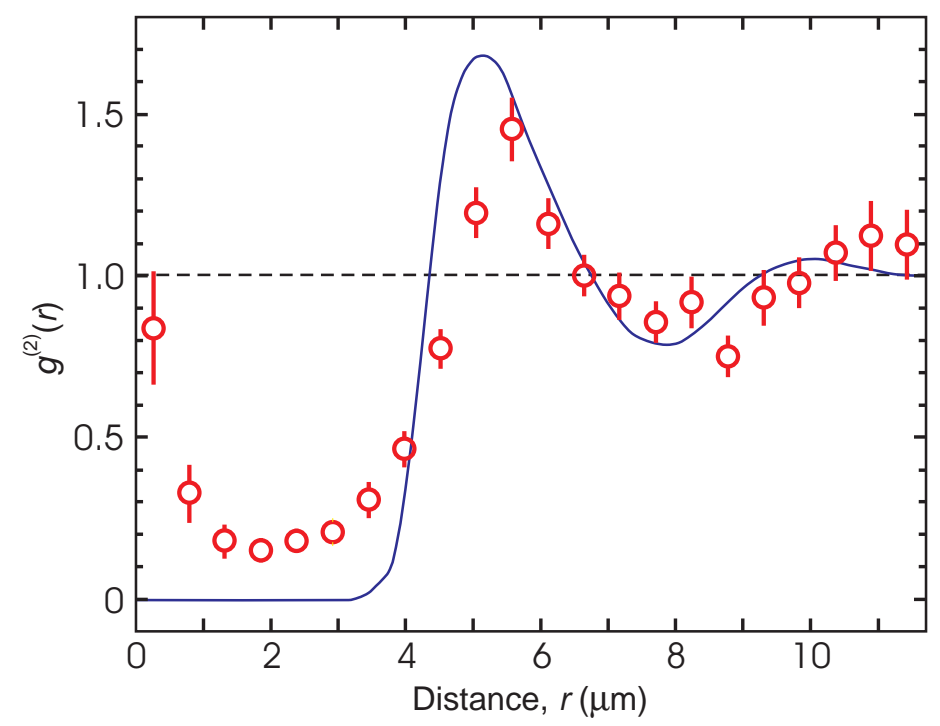

Figure 2. The pair correlation function of Rydberg atoms $g^{(2)}(r)$ depending on the distance $r$ between them: experimental data (red circles with error bars) and the standard theoretical prediction for the Rydberg blockade (blue solid curve). Adapted by permission from Macmillan Publishers Ltd: Nature, vol. 491, pp. 87-91, (C)2012.

It would be desirable, of course, to seek for the same peculiarity in other experiments with Rydberg blockade. Unfortunately, as far as we know, the other available highprecision installations, such as [13], cannot reach the required sub-micron range of distances.

To avoid misunderstanding, let us emphasize that formulas (37) and (39) can hardly be used to get the overall width of the unblocked zone, because it is actually composed of many unblocked sublevels whose positions are slightly shifted with respect to each other (see figure 1). So, this issue requires a more detailed treatment, which will be presented elsewhere.

Finally, it should be mentioned that yet another method for treating the Rydberg blockade in a nonuniform field might be based on the multi-polar expansion of Hamiltonian. The corresponding works - ranging from the detailed numerical calculations [14, 15] to the sophisticated analytical approaches [16]-were performed, first of all, in the context of excitation of the high-angular-momentum Rydberg states by single laser pulses. Unfortunately, since this technique was not applied by now to the problem of Rydberg blockade, we cannot make any comparison with the results of the present paper. Anyway, the fine spatial structure of the Rydberg blockade discussed in our previous work [8] appears already in the approximation of uniform inter-atomic field, and taking into account the spatial nonuniformity results only in quantitative modifications. 


\section{Conclusion}

In summary, we derived the exact general expression for Stark splitting of energy levels of a hydrogen-like atom by the nonuniform external electric field. The corresponding formula substantially refines the previously-known result in WKB approximation. Next, we used this expression for the multi-level treatment of the phenomenon of Rydberg blockade and obtained the characteristic parameters of the additional unblocked zones caused by the gradient term of the Stark splitting. It was found that the resulting positions of the unblocked zones are almost the same in the different limiting cases and, unexpectedly, coincide very well with the recent experimental measurements.

\section{Acknowledgments}

I am grateful to Profs. J-M Rost, R Côté and A V Gorshkov for fruitful discussions and advises. I am also grateful to the unknown referees for valuable bibliographic suggestions.

\section{References}

[1] Bethe H A and Salpeter E E 1957 Quantum Mechanics of One- and Two-Electron Atoms (New York: Academic Press)

[2] Gallagher T F 1994 Rydberg Atoms (Cambridge, UK: Cambridge Univ. Press)

[3] Bekenstein J D and Krieger J B 1970 Stark effect in hydrogen atoms for nonuniform fields. J. Math. Phys. 112721

[4] Lukin M D, Fleischhauer M, Cote R, Duan L M, Jaksch D, Cirac J I and Zoller P 2001 Dipole blockade and quantum information processing in mesoscopic atomic ensembles. Phys. Rev. Lett. 87037901

[5] Tong D, Farooqi S M, Stanojevic J, Krishnan S, Zhang Y P, Côté R, Eyler E E and Gould P L 2004 Local blockade of Rydberg excitation in an ultracold gas. Phys. Rev. Lett. 93063001

[6] Singer K, Reetz-Lamour M, Amthor T, Gustavo Marcassa L and Weidemüller M 2004 Suppression of excitation and spectral broadening induced by interactions in a cold gas of Rydberg atoms. Phys. Rev. Lett. 93163001

[7] Weidemüller M 2009 There can be only one. Nature Physics 5 91-2

[8] Dumin Y V 2014 Fine structure of the Rydberg blockade zone. J. Phys. B: At. Mol. Opt. Phys. 47175502

[9] Landau L D and Lifshitz E M 1977 Quantum Mechanics: Non-relativistic Theory (Course of Theoretical Physics, Vol. 3) 3rd ed (Oxford, UK: Pergamon Press)

[10] Silverstone H J 1978 Perturbation theory of the Stark effect in hydrogen to arbitrarily high order. Phys. Rev. A 18 1858-64

[11] Windholz L, Wasowicz T J, Drozdowski R and Kwela J 2012 Stark effect of atomic Helium singlet lines. J. Opt. Soc. Am. B 29 934-43

[12] Schauß P, Cheneau M, Endres M, Fukuhara T, Hild S, Omran A, Pohl T, Gross C, Kuhr S and Bloch I 2012 Observation of spatially ordered structures in a two-dimensional Rydberg gas. Nature $49187-91$

[13] Béguin L, Vernier A, Chicireanu R, Lahaye T and Browaeys A 2013 Direct measurement of the van der Waals interaction between two Rydberg atoms. Phys. Rev. Lett. 110263201

[14] Madsen L B, Hansen J R and Nilsen H M 1999 Procedures for the evaluation of atomic transition matrix elements in the interaction with laser light. Comput. Phys. Commun. 120 231-7 
[15] Komninos Y, Mercouris T and Nicolaides C A 2002 Theory and computation of the matrix elements of the full interaction of the electromagnetic field with an atomic state: Application to the Rydberg and the continuous spectrum. Phys. Rev. A 65043412

[16] Parzyński R and Sobczak M 2003 Beyond the long-wavelength approximation in electromagnetic Rydberg-Rydberg couplings. Opt. Commun. 225 123-30 